\title{
A serosurvey for anti-Burkholderiapseudomallei antibodies in peridomestic rats caught in rural farming and urban sites in Sri Lanka
}

\author{
CDGamage $^{1}$, DMuthusinghe ${ }^{1}$, YSarathkumara ${ }^{1}$, SGunawardana $^{2}$, MSRKhan $^{3}$, MHNorris $^{3}$, \\ ATuanyok $^{3}$
}

\section{Introduction}

The Sri Lankan National Melioidosis Surveillance Program has recently revealed nationwide distribution of invasive melioidosis that has placed Sri Lanka as one of the most endemic countries for melioidosis in South Asia. The causative bacterium is commonly acquired from the environment. There are no reported studies from Sri Lanka to identify exposure to $B$. pseudomallei in peridomestic animals. Thus, a serosurvey was conducted to determine the seroprevalence of anti-B. pseudomalle $i$ antibodies among peridomestic rats infesting an urban setting (Kandy) and a rural farming community (Girandurukotte) in Sri Lanka.

\section{Methods}

Rat (Rattusrattus) sera, collected under a research project of rodent-borne zoonotic diseases, were obtained from the Department of Microbiology, Faculty of Medicine, University of Peradeniya, Sri Lanka. Ninety two and 157 rat sera from Girandurukotte (GK), Badulla District and Kandy (KY), Kandy District respectively, were analyzed. Presence of anti-B. pseudomallei antibodies was established by an ELISA based on lipopolysaccharide (LPS) extracted from $B$. pseudomallei Bp82. The results were validated by Western Blot (WB) analysis with various $B$. pseudomallei LPS types and cell lysate. Cut-off values were determined statistically based on ELISA results.

\section{Results}

Of rat sera, $41(45 \%)$ from GK and 47 from KY (30\%) were seropositive by ELISA and confirmed by WB. In the WB assay, clear bands were shown to B. pseudomallei O-antigen type A and its cell lysate.

\section{Discussion and Conclusions}

The current study has shown that rats captured from both rural and urban sites have been exposed to B. pseudomallei or B. thailandensis since the B. pseudomallei O-antigen type A is known to be found in $B$. thailandensis, a non-pathogenic soil bacterium. However, $B$. thailandensis has not been reported in Sri Lanka. Thus, we recommend further in-depth analysis to confirm current findings.

\footnotetext{
${ }^{1}$ Department of Microbiology, Faculty of Medicine, University of Peradeniya, Sri Lanka

${ }^{2}$ Department of Animal Production and Health, Getambe, Sri Lanka

${ }^{3}$ Department of Infectious Diseases and Pathology, College of Veterinary Medicine \& Emerging Pathogens Institute, University of Florida, USA.

Address for correspondence: Dr ChandikaGamage, Department of Microbiology, Faculty of Medicine, University of Peradeniya, Sri Lanka. +94 771661460 Email:chandika@pdn.ac.lk https://orcid.org/0000-0003-0974-5730
} 